\title{
Real-world performance of blood-based proteomic profiling in first-line immunotherapy treatment in advanced stage non-small cell lung cancer
}

\author{
Patricia Rich, ${ }^{1}$ R Brian Mitchell, ${ }^{2}$ Eric Schaefer, ${ }^{3}$ Paul R Walker, ${ }^{4}$ John W Dubay, ${ }^{5}$ \\ Jason Boyd, ${ }^{6}$ David Oubre, ${ }^{7}$ Ray Page, ${ }^{8}$ Mazen Khalil, ${ }^{9}$ Suman Sinha, ${ }^{10}$ \\ Scott Boniol, ${ }^{11}$ Hafez Halawani, ${ }^{12}$ Edgardo S Santos, ${ }^{13}$ Warren Brenner, ${ }^{14}$ \\ James M Orsini, ${ }^{15}$ Emily Pauli, ${ }^{16}$ Jonathan Goldberg, ${ }^{17}$ Andrea Veatch, ${ }^{18}$ \\ Mitchell Haut, ${ }^{19}$ Bassam Ghabach, ${ }^{20}$ Savita Bidyasar, ${ }^{21}$ Maria Quejada, ${ }^{22}$ \\ Waseemullah Khan, ${ }^{23}$ Kan Huang, ${ }^{24}$ Linda Traylor, ${ }^{25}$ Wallace Akerley ${ }^{26}$
}

To cite: Rich $P$, Mitchell RB, Schaefer E, et al. Real-world performance of blood-based proteomic profiling in first-line immunotherapy treatment in advanced stage non-small cell lung cancer. Journal for ImmunoTherapy of Cancer 2021;9:e002989. doi:10.1136/ jitc-2021-002989

- Additional supplemental material is published online only. To view, please visit the journal online (http://dx.doi.org/10. 1136/jitc-2021-002989).

Some results have been presented at the 2020 American Society of Clinical Oncology Virtual Meeting.

Accepted 07 September 2021

Check for updates

(c) Author(s) (or their employer(s)) 2021. Re-use permitted under CC BY-NC. No commercial re-use. See rights and permissions. Published by BMJ.

For numbered affiliations see end of article.

Correspondence to Dr Wallace Akerley; Wallace.Akerley@hci.utah.edu

\section{ABSTRACT}

Purpose Immune checkpoint inhibition (ICI) therapy has improved patient outcomes in advanced non-small cell lung cancer (NSCLC), but better biomarkers are needed. A clinically validated, blood-based proteomic test, or host immune classifier (HIC), was assessed for its ability to predict ICI therapy outcomes in this real-world, prospectively designed, observational study.

Materials and methods The prospectively designed, observational registry study INSIGHT (Clinical Effectiveness Assessment of VeriStrat $\circledast$ Testing and Validation of Immunotherapy Tests in NSCLC Subjects) (NCT03289780) includes 35 US sites having enrolled over 3570 NSCLC patients at any stage and line of therapy. After enrolment and prior to therapy initiation, all patients are tested and designated HIC-Hot (HIC-H) or HIC-Cold (HIC-C). A prespecified interim analysis was performed after 1-year follow-up with the first 2000 enrolled patients. We report the overall survival (OS) of patients with advanced stage (IIIB and IV) NSCLC treated in the first-line (ICl-containing therapies $n=284$; all first-line therapies $n=877$ ), by treatment type and in HIC-defined subgroups. Results OS for HIC-H patients was longer than OS for HIC-C patients across treatment regimens, including $\mathrm{ICl}$. For patients treated with all ICl regimens, median OS was not reached ( $95 \% \mathrm{Cl} 15.4$ to undefined months) for HIC-H ( $n=196)$ vs 5.0 months $(95 \% \mathrm{Cl} 2.9$ to 6.4$)$ for HIC-C patients $(n=88) ; \mathrm{HR}=0.38(95 \% \mathrm{Cl} 0.27$ to 0.53$)$, $\mathrm{p}<0.0001$. For ICI monotherapy, OS was 16.8 vs 2.8 months ( $\mathrm{HR}=0.36$ (95\% $\mathrm{Cl} 0.22$ to 0.58$), \mathrm{p}<0.0001)$ and for ICl with chemotherapy OS was unreached vs 6.4 months ( $\mathrm{HR}=0.41$ (95\% $\mathrm{Cl} 0.26$ to 0.67), $\mathrm{p}=0.0003)$. HIC results were independent of programmed death ligand 1 (PD-L1). In a subgroup with PD-L1 $\geq 50 \%$ and performance status $0-1$, HIC stratified survival significantly for ICI monotherapy but not ICl with chemotherapy. Conclusion Blood-based HIC proteomic testing provides clinically meaningful information for immunotherapy treatment decision in NSCLC independent of PD-L1. The data suggest that HIC-C patients should not be treated with ICl alone regardless of their PD-L1 expression.

\section{INTRODUCTION}

Lung cancer is the leading cause of cancer death in the USA, but long-term survival is improving with new biomarker-directed therapies. ${ }^{12}$ Immune checkpoint inhibition (ICI) via blockade of the programmed death 1 (PD-1) axis-approved by the US Food and Drug Administration for lung cancer in 2015-has slowed tumor progression, produced durable responses, and improved overall survival (OS) in many patients with non-small cell lung cancer (NSCLC). ${ }^{3}$ ICI alone or in combination with chemotherapy has become standard of care first-line treatment for NSCLC patients without an actionable mutation. ICI monotherapy is indicated for tumor PD ligand 1 (PD-L1) expression $>50 \%$ (optional for PD-L1 >1\%) and ICI with chemotherapy for all PD-L1 expression levels. $^{45}$

Tumor PD-L1 is the recommended biomarker for ICI monotherapy eligibility in NSCLC, ${ }^{6}$ although only $~ 30 \%$ of patients with PD-L1 $\geq 50 \%$ achieve 5 -year survival. ${ }^{78}$ Conversely, some patients with PD-L1 negative cancers have responded to first-line ICI monotherapies but are ineligible for first-line ICI monotherapy under current guidelines. ${ }^{6}$ Considering the inconsistent correlation between PD-L1 expression and ICI efficacy, the significant adverse events profile of ICI $^{4}$ and their cost to the patient and the healthcare system, ${ }^{9}$ finding additional indicators of patient benefit from ICI combinations or monotherapy is a focus for both physicians and patients.

Predictive and prognostic tests that aid therapeutic decision making are critical for 
optimizing patient outcomes while minimizing toxicity and costs. A pretreatment blood-based proteomic test, termed a host immune classifier (HIC), was developed in a multi-institutional, correlative study of clinical outcomes in patients with NSCLC. ${ }^{10}$ Using matrix-assisted laser desorption/ionization time-of-flight mass spectrometry combined with a basic machine learning algorithm, HIC defines a proteomic classification broadly prognostic of outcomes in NSCLC. Patients classified as HIC-Hot (HIC-H) have an increased likelihood of better outcomes across multiple therapies and lines of therapy, often surviving 2-3 times longer than patients classified as HICCold (HIC-C)..$^{11-13}$

Studies of the underlying biology captured by the HIC test have shown it to be a measure of systemic inflammatory state, deriving classifications from a proteomic signature comprizing isoforms of serum amyloid A1 ${ }^{14}$ Recent work has shown that, in general, all eight mass spectral features used in the HIC test algorithm play a role in generation of the test result and that their relative importance varies from one patient to another. ${ }^{15}$ Increased levels of proinflammatory proteins, including $\mathrm{C}$ reactive protein, interleukin-6, serum amyloid A, cytokeratin 19 fragment (CYFRA 21-1), insulin-like growth factor (IGF)II, osteopontin, and ferritin, have been observed in the serum collected from HIC-C patients. ${ }^{16}$ Set enrichment and pathway analyses have indicated association of HIC test classification with acute phase response, ${ }^{16-18}$ acute inflammatory response, and complement activation. ${ }^{17} 18$ These factors have been shown to be correlated with a dysfunctional tumor immune response and poor prognosis in general and in the context of immunotherapy. ${ }^{19-22}$

The INSIGHT (Clinical Effectiveness Assessment of VeriStrat ${ }^{\circledR}$ Testing and Validation of Immunotherapy Tests in NSCLC Subjects) study (NCT03289780) is an ongoing, observational registry trial, designed to validate that HIC test classifications stratify clinical outcomes for real-world patients receiving standard of care therapies, including immunotherapy. ${ }^{23}$ Although extensively evaluated in patients receiving targeted therapy and chemotherapy-based regimens, HIC's clinical utility for patients treated with ICI has been demonstrated in only one previous study. ${ }^{24}$ Here, we report results of a prespecified interim analysis of INSIGHT assessing HIC's stratification of survival in the first 2000 enrolled patients with at least 1 year of follow-up, focusing on patients with advanced NSCLC treated in the first-line with platinum chemotherapy and ICI-containing regimens.

\section{MATERIALS AND METHODS}

\section{Study design and patient enrolment}

Since April 4, 2016, INSIGHT has enrolled over 3570 patients from 35 different US sites, including all stages of NSCLC (stage I-IV), histology, and Eastern Cooperative Oncology Group (ECOG) performance status (PS). Enrolled patients had a diagnosis of NSCLC, were 18 years or older, had a tumor identified as epidermal growth factor receptor (EGFR) wild type or unknown, and underwent HIC testing. Patients with unknown EGFR mutation status were subject to plasma-based mutation testing ${ }^{25}$ and patients positive for EGFR mutations were excluded from the study. Patients were followed for outcome (OS for all patients and time to recurrence for early stage patients) for 18 months.

This interim analysis of secondary and exploratory endpoints involving the HIC test was carried out after at least 12 months follow-up of the first 2000 enrolled subjects. The Analysis Population was defined as all enrolled patients with at least 12 months follow-up, HIC classification of Hot or Cold, and available data on treatment received.

\section{Biomarker testing}

All patients received HIC testing (VeriStrat ${ }^{\circledR}$ proteomic test, Biodesix, Boulder, Colorado, USA). Venous blood was collected at the time of study enrolment and prior to the start of treatment. All HIC testing was performed in a centralized clinical laboratory (Biodesix), Accredited by Clinical Laboratory Improvement Amendments/College of American Pathologists and Approved by New York State Clinical Laboratory Evaluation Program. Serum samples for HIC testing were collected and processed as previously described. ${ }^{10}{ }^{12}$ Spectra from each sample were collected and processed in triplicate. When all three spectra produced the same classification, this was reported as the HIC test result-(HIC-H, aka VeriStrat Good) or (HIC-C, aka VeriStrat Poor).

PD-L1 expression was collected from physicians when possible. Results from any PD-L1 tissue-based assay of physician choice were admissible.

\section{Statistical analysis}

All statistical analyses, including covariates to be used in multivariate analyses and all subgroups for analysis, were prespecified in the Statistical Analysis Plan prior to interim analysis database lock. OS was defined as time between patient enrolment in the study and date of death or censored at date of last follow-up. OS was summarized within patient subgroups by its median and $95 \%$ CI and compared between patient subgroups by Cox proportional HRs, their CIs, and corresponding $\mathrm{p}$ values. Statistical analyses were carried out using JMP V.15.1.0 (SAS Institute) or PRISM (GraphPad, La Jolla, California, USA). Association of baseline characteristics between patient subgroups was assessed using Mann-Whitney U test (with t-test approximation) for continuous attributes and Fisher's exact test or $\chi^{2}$ test for categorical attributes.

\section{RESULTS}

\section{Patient inclusion and demographics}

Between study launch in April 2016 and database lock on March 15, 2020, INSIGHT had accrued 1975 evaluable subjects (figure 1). Of these, 1464 had advanced stages of NSCLC (stage IIIB or stage IV), with 877 having had no 
Patients enrolled in INSIGHT study to date $(n=3726)$
Excluded patients who were enrolled since data cutoff $(n=570)$

Patients enrolled in INSIGHT study at time of data cutoff $(n=3000)$

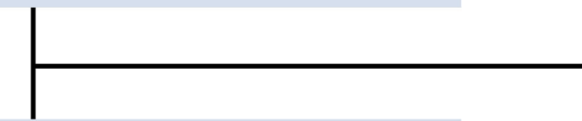

Patients with $\geq 1$-year follow-up $(n=2376)$

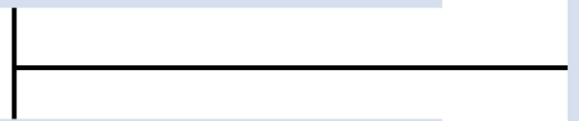

Patients in the Analysis Population ( $n=1975$ )
Excluded patients with < 1-year follow-up $(n=624)$

Excluded $(n=401)$ :

- Not eligible and screen fails ( $n=198)$

- Patients with incomplete records ( $n=163$ )

- Indeterminate HIC status $(n=40)$

Patients with stage IIIB-IV NSCLC ( $n=1464)$

Patients with stage I-IIIA NSCLC $(n=511)$

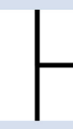

Patients with no prior therapy for advanced disease $(n=877)$ :

- Patients receiving $\mathrm{ICl}$-containing regimens ( $n=284$ )

- Patients receiving platinum-doublet based chemotherapy (no ICI) ( $n=392)$

- Patients receiving other therapies (no ICl) ( $n=201$ )

Figure 1 Disposition of patients in the study interim analysis. HIC, host immune classifier; ICI, immune checkpoint inhibitor; NSCLC, non-small cell lung cancer.

prior therapy for advanced disease (ie, receiving first-line therapy in this study). A total of 587 patients enrolled at second and higher lines of therapies.

The baseline characteristics of the patients in the Analysis Population who moreover had advanced disease and no prior therapy $(\mathrm{n}=877)$ are summarized in table 1 . Average age was 68 years in this subgroup. Patients primarily had adenocarcinoma histology (65.3\%) followed by squamous cell carcinoma (21.4\%); the remainder $(13.2 \%)$ were not specified beyond NSCLC or had an uncommon histology. ECOG PS 1 was the most common status at the time of enrolment although $25.4 \%$ of patients enrolled had ECOG PS>1 (table 1). A total of $91 \%$ of patients had metastases, including $18.5 \%$ with brain metastases (online supplemental table S1).

\section{Treatment regimens}

Treatment choice was left to physician discretion. Therefore, various standard of care treatments were represented, including platinum-based therapy (ie, carboplatin or cisplatin in combination with pemetrexed or paclitaxel), ICI (monotherapy or in combination with chemotherapy), non-platinum chemotherapy, tyrosine kinase inhibitors, radiation, surgery or best supportive care for those with no active treatment (table 1). The two most common types of first-line therapies used in this study were platinum-based chemotherapy $(n=392$, $44.7 \%)$ and ICI in combination with other agents $(n=167$, $19.0 \%)$, primarily chemotherapy $(\mathrm{n}=161,18.4 \%)$.

\section{Patient outcomes by treatment regimen}

The median OS (mOS) of advanced stage patients treated with first-line platinum-based chemotherapy was 11.7 (95\% CI 9.9 to 14.7) months (figure 2A). Patients treated with ICI (either monotherapy or ICI in combination with other agents) demonstrated an mOS of 14.4 (95\% CI 10.0 to undefined) months. 
Table 1 Patient demographics and clinical characteristics by HIC classification $(n=877)$

\begin{tabular}{|c|c|c|c|c|}
\hline & $\begin{array}{l}\begin{array}{l}\text { Total } \\
(\mathrm{n}=877)\end{array} \\
\end{array}$ & $\begin{array}{l}\text { HIC-hot } \\
(n=622,70.9 \%)\end{array}$ & $\begin{array}{l}\text { HIC-cold } \\
(\mathrm{n}=255,29.1 \%)\end{array}$ & $P$ value \\
\hline \multicolumn{5}{|l|}{ Age } \\
\hline Mean (SD) & $68.0(10.2)$ & $68.4(10.3)$ & $67.0(10.0)$ & 0.070 \\
\hline Median (range) & $67.6(35.2-95.1)$ & $68.1(35.2-92.9)$ & $66.5(36.5-95.1)$ & 0.075 \\
\hline Gender, n (\%) & & & & 0.004 \\
\hline Female & $382(43.6)$ & $290(46.6)$ & $92(36.1)$ & \\
\hline Male & $495(56.4)$ & $332(53.4)$ & $163(63.9)$ & \\
\hline Histology, n (\%) & & & & $<0.001$ \\
\hline Adenocarcinoma & $573(65.3)$ & $430(69.1)$ & $143(56.1)$ & \\
\hline Squamous & $188(21.4)$ & $116(18.6)$ & $72(28.2)$ & \\
\hline NSCLC other & $116(13.2)$ & $76(12.2)$ & $40(15.7)$ & \\
\hline \multicolumn{2}{|c|}{ Disease stage at study entry, $\mathrm{n}(\%)$} & & & 0.739 \\
\hline Stage IIIB & $112(12.8)$ & $78(12.5)$ & $34(13.3)$ & \\
\hline Stage IV & $765(87.2)$ & $544(87.5)$ & $221(86.7)$ & \\
\hline \multicolumn{2}{|c|}{ ECOG status at study entry, $\mathrm{n}(\%)$} & & & $<0.001$ \\
\hline PS 0 & $222(25.3)$ & $184(29.6)$ & $38(14.9)$ & \\
\hline PS 1 & $432(49.3)$ & $299(48.1)$ & $133(52.2)$ & \\
\hline PS 2 & $183(20.9)$ & $117(18.8)$ & $66(25.9)$ & \\
\hline PS 3 & $35(4.0)$ & $22(3.5)$ & $13(5.1)$ & \\
\hline PS 4 & $5(0.6)$ & $0(0.0)$ & $5(2.0)$ & \\
\hline \multicolumn{2}{|l|}{ Smoking status, n (\%) } & & & 0.056 \\
\hline Current smoker & $332(37.9)$ & $230(37.0)$ & $102(40.0)$ & \\
\hline Former smoker & $441(50.3)$ & $308(49.5)$ & $133(52.2)$ & \\
\hline Never smoker & $104(11.9)$ & $84(13.5)$ & $20(7.8)$ & \\
\hline \multicolumn{2}{|l|}{ PD-L1 expression, n (\%) } & & & 0.632 \\
\hline High ( $\geq 50 \%)$ & $168(19.2)$ & $119(19.1)$ & 49 (19.2) & \\
\hline Low ( $\geq 1 \%$ and $<50 \%)$ & $137(15.6)$ & $100(16.1)$ & $37(14.5)$ & \\
\hline Negative (<1\%) & $169(19.3)$ & $125(20.1)$ & $44(17.3)$ & \\
\hline NA & $403(46.0)$ & $278(44.7)$ & $125(49.0)$ & \\
\hline \multicolumn{2}{|c|}{ Disease status at study entry, $\mathrm{n}(\%)$} & & & $<0.001$ \\
\hline Newly diagnosed & $820(93.5)$ & $571(91.8)$ & $249(97.6)$ & \\
\hline Recurrent & $55(6.3)$ & $50(8.0)$ & $5(2.0)$ & \\
\hline Other & $2(0.2)$ & $1(0.2)$ & $1(0.4)$ & \\
\hline \multicolumn{2}{|l|}{ Treatment regimen, $\mathrm{n}(\%)$} & & & 0.026 \\
\hline $\mathrm{ICl}$ monotherapy & $117(13.3)$ & $80(12.9)$ & $37(14.5)$ & \\
\hline $\mathrm{ICl}+$ chemotherapy & $161(18.4)$ & $113(18.2)$ & $48(18.8)$ & \\
\hline $\mathrm{ICl}$ other combination & $6(0.7)$ & $3(0.5)$ & $3(1.2)$ & \\
\hline Pt-based chemotherapy & $392(44.7)$ & $284(45.7)$ & $108(42.4)$ & \\
\hline Other chemotherapy & $19(2.2)$ & $13(2.1)$ & $6(2.4)$ & \\
\hline TKI & $40(4.6)$ & $35(5.6)$ & $5(2.0)$ & \\
\hline Radiation & $32(3.6)$ & $25(4.0)$ & $7(2.7)$ & \\
\hline Other active treatment & $16(1.8)$ & $14(2.3)$ & $2(0.8)$ & \\
\hline No active treatment & $94(10.7)$ & $55(8.8)$ & 39 (15.3) & \\
\hline
\end{tabular}

ECOG, Eastern Cooperative Oncology Group; HIC, host immune classifier; ICI, immune checkpoint inhibition; NA, not available; NSCLC, nonsmall cell lung cancer; PD-L1, programmed death ligand 1; PS, performance status; Pt, platinum; TKI, tyrosine kinase inhibitor. 
A
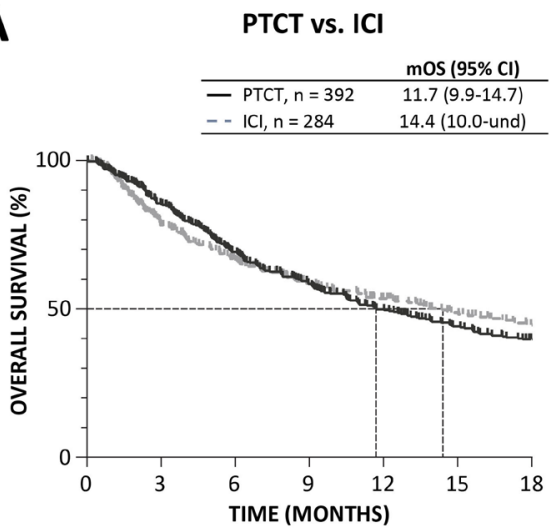

No. at Risk:

$\begin{array}{lllllll}\text { PTCT 392 } & 318 & 249 & 206 & 167 & 143 & 79\end{array}$
B

B

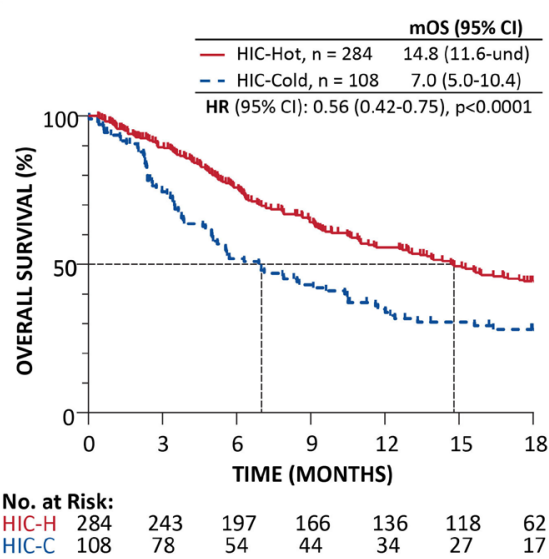

C ICI by HIC

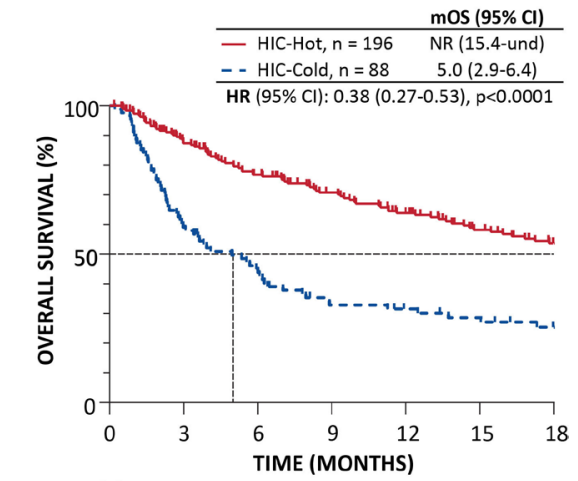

No. at Risk:

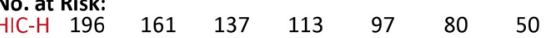

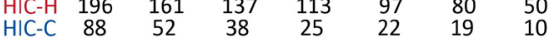

Figure 2 Kalpan-Meier analysis of overall survival in patients with advanced stage NSCLC. (A) Received platinum-doublet chemotherapy (PTCT) or ICl-containing therapy (ICl) in the first-line setting, and in subsets of patients receiving (B) PTCT and (C) ICl-containing regimen by $\mathrm{HIC}$ classification. HIC-C, host immune classifier-cold; $\mathrm{HIC}-\mathrm{H}$, $\mathrm{HIC}$-hot; ICl, immune checkpoint inhibition; mOS, median overall survival in months; NR, not reached; NSCLC, non-small cell lung cancer; und, undefined.

\section{HIC classification and OS}

In the advanced stage first-line population, $71 \%$ of subjects were classified as HIC-H and 29\% were classified as HIC-C (table 1), consistent with previous reports. ${ }^{10} 111326$ Between HIC-H and HIC-C groups, there were no statistically significant differences in age, disease stage at entry, ECOG PS, smoking status, or PD-L1 expression. However, statistically significant differences were observed in gender, histology, and treatment regimen.

The OS of patients treated with first-line platinumbased chemotherapy and ICI regimens was further stratified using the HIC. In both treatment groups, patients classified as HIC-H had significantly longer OS than patients classified as HIC-C (figure 2B,C). The mOS of HIC-H patients treated with platinum-based chemotherapy was 14.8 months compared with 7.0 months for HIC-C patients (HR=0.56 (95\% CI 0.42 to 0.75$)$, $\mathrm{p}<0.0001$ ) (figure 2B). For all patients treated with ICI regimens (monotherapy or combination therapies), mOS was not reached for HIC-H patients compared with 5.0 months for HIC-C patients (HR=0.38 (95\% CI 0.27 to 0.53 ), $\mathrm{p}<0.0001$ ) (figure $2 \mathrm{C}$ ). Patients not receiving an active therapy classified as HIC-H also had longer survival than HIC-C, at 4.8 months compared with 1.6 ( $\mathrm{HR}=0.43$ (95\% CI 0.26 to 0.73 ), $\mathrm{p}=0.002$ ) (online supplemental figure $\mathrm{S} 1)$.

In the subgroup treated with ICI monotherapy, patients classified as HIC-H had mOS 16.8 months, whereas mOS in patients classified as HIC-C was 2.8 months (80/37 HIC-H/HIC-C, HR=0.36 (95\% CI 0.22 to 0.58), $\mathrm{p}<0.0001$ ) (figure $3 \mathrm{~A}$ ). When patients were treated with first-line ICI combined with chemotherapy, patients classified as HIC-H had significantly longer mOS compared with patients classified as HIC-C (113/48 HIC-H/HIC-C, median not reached vs 6.4 months, HR $=0.41$ (95\% CI 0.26 to 0.67 ), $\mathrm{p}<0.001$ ) (figure $3 \mathrm{~B}$ ).

\section{HIC and PD-L1 expression}

Among patients treated with ICI, no statistically significant differences appeared in the proportions of HIC-H and HIC-C patients at each PD-L1 expression level analyzed (table 2). HIC is therefore independent of PD-L1 expression. As expected, survival was longer in the PD-L1 High subgroup than PD-L1 Low or Negative, although few PD-L1 Low or Negative patients received ICI monotherapy (figure 3C,D). With the PD-L1 Low and Negative groups (PD-L1 <50\%) combined and the PD-L1 not available (NA) group included, survival was significantly longer for PD-L1 NA compared with PD-L1 $<50 \%$ but not different from PD-L $1 \geq 50 \%$ in the group receiving ICI with chemotherapy, and no differences appeared in the ICI monotherapy group (online supplemental figure S2). In the PD-L1 High, PS 0-1 subgroup receiving ICI monotherapy, subjects classified as HIC-H had significantly longer OS, mOS not reached compared with 2.6 months for HIC-C $(\mathrm{HR}=0.22$ (95\% CI 0.10 to 0.48$), \mathrm{p}<0.001)$ (figure $3 \mathrm{E}$ ). However, for subjects receiving ICI with chemotherapy in the PD-L1 High subgroup, mOS was not reached for both HIC-H and HIC-C patients ( $\mathrm{HR}=0.77$ (95\% CI 0.25 to 2.35 ), $\mathrm{p}=0.641$ ) (figure $3 \mathrm{~F}$ ).

In a multivariate analysis, HIC was identified as an independent predictor of $\mathrm{OS}$ of ICI-treated patients ( $\mathrm{HR}=0.37$ (95\% CI 0.25 to 0.53$), \mathrm{p}<0.0001)$, when adjusted for demographic characteristics such as gender, ECOG PS, histology, age, disease stage, and PD-L1 expression (table 3). Multivariate analysis further indicated that ECOG PS, age, and histology were significantly associated with survival to a lesser extent, that patients receiving ICI + chemotherapy fared better than patients receiving ICI monotherapy, and that patients with high PD-L1 expression fared better than those that were PD-L1 negative but not necessarily those with low PD-L1 expression. Smoking status, stage, and gender were not significantly associated with survival. Limiting the analysis to the 
A

ICI Monotherapy by HIC

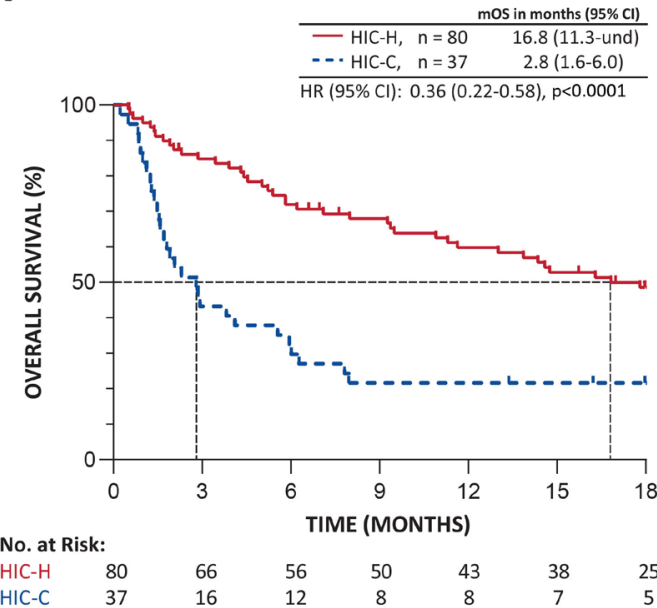

C

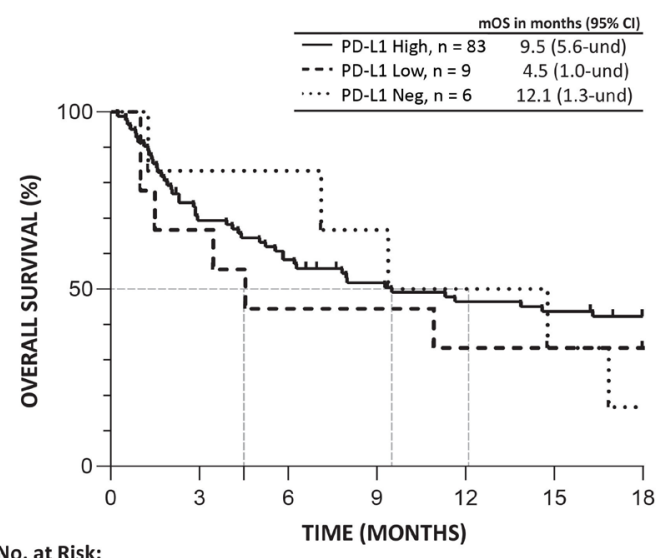

$\begin{array}{lllllll}\text { PD-L1 High } 83 & 56 & 47 & 39 & 34 & 32 & 22\end{array}$ $\begin{array}{cccccccc}\text { PD-L1 Low } & 9 & 6 & 4 & 4 & 3 & 3 & 2 \\ \text { PD-L1 Neg } & 6 & 5 & 5 & 4 & 3 & 2 & 1\end{array}$

$\mathbf{E}$

PD-L1 High, PS 0-1: ICI Monotherapy by HIC

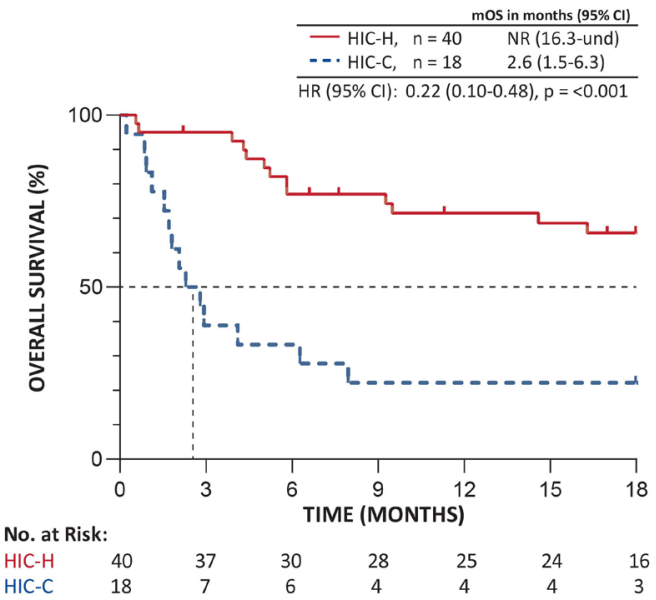

B

$\mathrm{ICI}+$ Chemotherapy by HIC

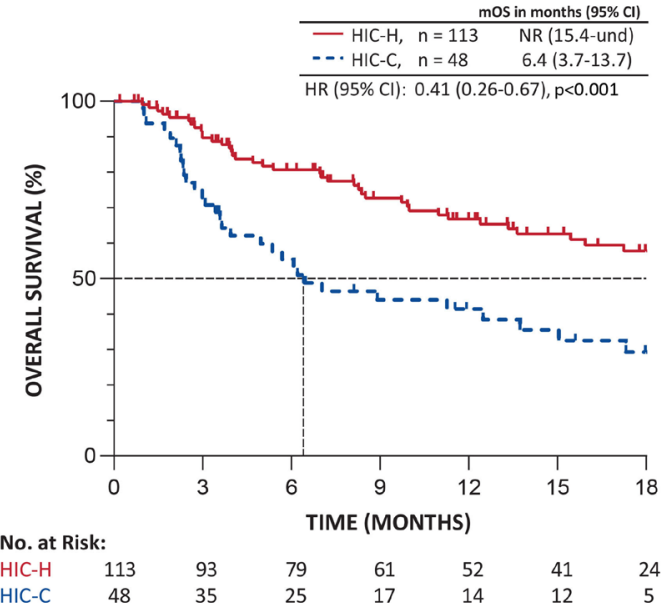

D

$\mathrm{ICl}+$ Chemotherapy by PD-L1

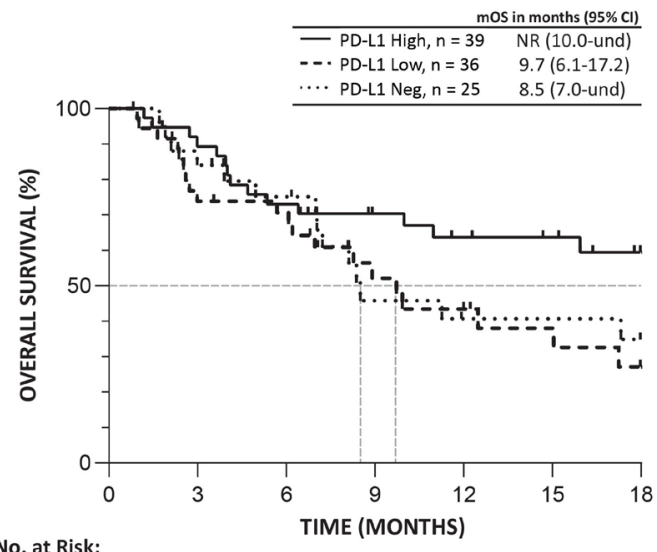

$\begin{array}{llllllll}\text { PD-L1 High } & 39 & 33 & 27 & 21 & 18 & 16 & 8\end{array}$ $\begin{array}{llllclll}\text { PD-L1 Low } & 36 & 24 & 22 & 12 & 9 & 7 & 4 \\ \text { PD-L1 Neg } & 25 & 21 & 17 & 9 & 7 & 7 & 3\end{array}$

$\mathbf{F}$

PD-L1 High, PS 0-1: ICI + Chemotherapy by HIC
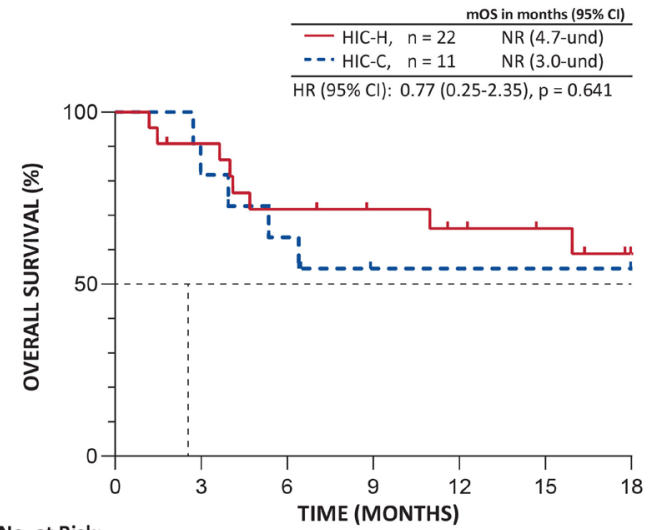

No. at Risk:

$\begin{array}{llcccccc}\text { HIC-H } & 22 & 19 & 15 & 13 & 11 & 9 & 4 \\ \text { HIC-C } & 11 & 9 & 7 & 4 & 4 & 4 & 2\end{array}$

Figure 3 Kaplan-Meier analysis of overall survival in patients with advanced stage NSCLC receiving (A, C, E) ICl as monotherapy or (B, D, F) ICl in combination with chemotherapy by (A, B) HIC classification, (C, D) PD-L1 expression (omitting PD-L1 status NA), and (E, F) by HIC classification in the subsets of patients with high PD-L1 expression and ECOG PS 0-1. PD-L1 high, PD-L1 expression $\geq 50 \%$; PD-L1 low, PD-L1 $\geq 1 \%$ and $<50 \%$; PD-L1 neg, PD-L1 negative ( $<1 \%)$; und, undefined. ECOG, Eastern Cooperative Oncology Group; HIC, host immune classifier; HIC-C, HIC-cold; HIC-H, HIC-hot; ICl, immune checkpoint inhibition; mOS, median overall survival; NA, not available; NR, not reached; NSCLC, non-small cell lung cancer; PD-L1, programmed death ligand 1; PS, performance status. 
Table 2 PD-L1 expression of ICl-receiving subjects by HIC classification

\begin{tabular}{llll}
\hline $\begin{array}{l}\text { PD-L1 expression, } \\
\mathbf{n}(\%)\end{array}$ & $\begin{array}{l}\text { HIC-H } \\
(\mathbf{n}=\mathbf{1 3 4 )}\end{array}$ & $\begin{array}{l}\text { HIC-C } \\
\mathbf{( n = 6 7 )}\end{array}$ & $\begin{array}{l}\text { P value } \\
\text { by } \chi^{2}\end{array}$ \\
\hline Negative $(<1)$ & $21(16 \%)$ & $12(18 \%)$ & 0.854 \\
Low $(\geq 1 \%$ and $<50)$ & $32(24 \%)$ & $14(21 \%)$ & \\
High $(\geq 50)$ & $81(60 \%)$ & $41(61 \%)$ & \\
\hline
\end{tabular}

$\mathrm{HIC}$, host immune classifier; HIC-C, HIC-cold; HIC-H, HIC-hot; ICI, immune checkpoint inhibition; PD-L1, programmed death ligand 1.

ECOG PS 0-1, PD-L1 High subgroup balanced the covariates (online supplemental tables 2-4) and maintained $\mathrm{HIC}$ as a predictor of survival along with treatment type. In the ECOG PS 0-1, PD-L1 High subgroup, patients classified as HIC-H fared similarly whether treated with ICI monotherapy or ICI + chemotherapy, whereas those classified as HIC-C fared significantly better with ICI + chemotherapy (online supplemental figure S4).

\section{DISCUSSION}

This interim analysis of INSIGHT evaluated immunotherapy and chemotherapy regimen performances overall and in populations stratified by a proteomic test. Clinical observations that stand out are (1) the extremely poor survival of HIC-C patients given ICI alone or no active treatment, (2) the independent prognostic significance of HIC in the setting of immunotherapy and (3) the independence of HIC and PD-L1 in predicting survival with ICI-based therapy. As more patients participate in this actively enrolling study, the performance of ICI regimens will be continually evaluated to see if these data hold and if patients can be further subdivided by other clinical factors and biomarkers physicians use to make treatment decisions. Nevertheless, these data emphasize the need for improved biomarkers that not only identify expression of a drug target, but fully and objectively characterize the patient's disease state. ${ }^{27}$

Interestingly, in this study, mOS for patients treated with ICI regimens overlapped with mOS for patients treated with platinum-based chemotherapy. Though seemingly at odds with previously published randomized clinical trial (RCT) results, the duration of mOS observed on ICI therapy here is consistent with other real-world studies. ${ }^{28-30}$ Moreover, it is worth noting that this is a summary result rather than a direct comparison between therapies, and could be due to the observational nature of this trial, the fact that patients were not randomized but rather therapy was decided by their physicians based on a multitude of factors including HIC results, ${ }^{31}$ changes in treatment paradigms over the course of the study, and the inclusion of a heterogeneous real-world patient population often excluded from RCTs.

It is significant that this study enrolls a real-world patient population. Though discrepancy between RCT results and those obtained in community practice is a well-known phenomenon, its scope and impact on patient conversations is neither fully appreciated nor openly discussed. In particular, the prescriptive patient populations enrolled in RCTs may not be representative of an everyday lung cancer patient in real-world practice. Of note, ECOG PS is known to be a significant factor in patient response to therapy, yet RCTs nearly always exclude patients with

\begin{tabular}{|c|c|c|c|}
\hline Covariate & & HR $(95 \% \mathrm{Cl})$ & $P$ value \\
\hline HIC (vs HIC-cold) & HIC-hot & $0.37(0.25$ to 0.53$)$ & $<0.0001$ \\
\hline \multirow[t]{2}{*}{ ECOG (vs PS 0) } & PS 1 & 1.47 (0.89 to 2.42$)$ & 0.133 \\
\hline & PS 2+ & 2.64 (1.53 to 4.54$)$ & $<0.001$ \\
\hline Age (vs <65 years) & $\geq 65$ years & 1.52 (1.07 to 2.15$)$ & 0.019 \\
\hline \multirow[t]{2}{*}{ Histology (vs adenocarcinoma) } & Squamous cell & 1.45 (0.87 to 2.39$)$ & 0.151 \\
\hline & NSCLC other & $0.54(0.30$ to 0.99$)$ & 0.045 \\
\hline \multirow[t]{2}{*}{ Therapy (vs ICl+ chemotherapy) } & $\mathrm{ICl}$ monotherapy & 1.67 (1.11 to 2.51$)$ & 0.013 \\
\hline & $\mathrm{ICl}$ with other & 1.94 (0.66 to 5.74$)$ & 0.230 \\
\hline \multirow[t]{3}{*}{ PD-L1 expression (vs high ( $\geq 50 \%)$ ) } & PD-L1 low ( $\geq 1 \%$ and $<50 \%)$ & 1.54 (0.91 to 2.60$)$ & 0.109 \\
\hline & PD-L1 negative (<1\%) & 1.88 (1.07 to 3.32$)$ & 0.028 \\
\hline & $\mathrm{N} / \mathrm{A}$ & 0.99 (0.62 to 1.61$)$ & 0.980 \\
\hline Smoking history (vs never) & Ever & 1.87 (0.89 to 3.91$)$ & 0.098 \\
\hline Disease stage (vs IIIB) & Stage IV & $1.50(0.72$ to 3.15$)$ & 0.281 \\
\hline Gender (vs female) & Male & 1.02 (0.71 to 1.46$)$ & 0.920 \\
\hline
\end{tabular}

Bold indicates parameter of highest significance.

$\mathrm{Cl}$, confidence interval; ECOG, Eastern Cooperative Oncology Group; HIC, host immune classifier; HR, hazard ratio; ICl, immune checkpoint inhibition; N/A, not available; NSCLC, non-small cell lung cancer; OS, overall survival; PD-L1, programmed death ligand 1 ; PS, performance status. 
worse ECOG PS who represent a large fraction of the broader patient population. Most if not all ICI clinical trials in NSCLC have restricted enrolment to PS $0-1 .{ }^{32-36}$ By contrast, in INSIGHT $25.4 \%$ of this interim analysis population had an ECOG PS of 2 or greater. RCTs also often exclude patients presenting with comorbid diseases, unstable disease, abnormal bloodwork, brain metastases, oxygen use, etc, which may be present in real-world populations, impacting tolerability and response to therapy. These study results, therefore, have potential to bridge the gap between RCTs and the broader NSCLC patient community, appropriately informing expectations and facilitating dialog between patients and care teams navigating the treatment landscape.

The prognostic significance of HIC classification in the setting of immunotherapy is noteworthy. While PD-L1 is viewed as the standard biomarker for PD-1-based immunotherapy for lung cancer, these data add to the body of evidence that checkpoint pathways represent a network that involves many other elements besides the PD-1/ PD-L1 complex. In multivariate analysis, PD-L1 alongside other conventional prognostic host factors, including age and histology, were only modestly prognostic of survival in this setting. Other conventional prognostic factors, including smoking status, stage, and gender, were not significantly prognostic of survival. Treatment with ICI + chemotherapy was significantly associated with better survival compared with treatment with ICI monotherapy in this analysis, which, to our knowledge, has not previously been demonstrated in a real-world, prospectively designed, retrospective analysis. This may have been due to treating physicians prescribing ICI alone for patients too ill for combination therapy. However, recent network meta-analyses of clinical trial results indicate ICI + chemotherapy improved OS numerically though not significantly over ICI monotherapy. ${ }^{37-39}$ Clinical trials directly comparing both treatments are ongoing. Notably, ECOG PS and HIC status were the most significant prognostic factors. These data suggest that PD-1/PD-L1 therapy is affected by host immune response extrinsic to the PD-1/ PD-L1 pathway, which is measured by the HIC. The relationship between HIC and a diseased inflammatory response related to PD-1/PD-L1 therapy has been noted previously, ${ }^{24}$ supporting systemic host protumor inflammatory processes as a resistance mechanism to PD-1/ PD-L1 therapy. ${ }^{40} 41$

It is noteworthy that for patients in the PD-L1 High subgroup, patients with a HIC-H classification had similar survival whether they were treated with ICI monotherapy or ICI + chemotherapy, whereas those with HIC-C classification had better survival on ICI + chemotherapy. This indicates that HIC identifies an aggressive disease state less responsive to ICI alone.

Due to the observational nature of this study, prognostic use of HIC within ICI treatment groups is limited by lack of controls, allowing for biases in covariates between the groups with a potential impact on the predictive analyses and claims. Reducing the ICI treatment group to PD-L1
High, ECOG PS 0-1 reduces the potential biases in these covariates and creates more balanced and comparable groups. While the populations are smaller, this subgroup resembles more closely the controls of a randomized trial in which predictive interactions can be measured. This result suggests that controlled trials may miss important patient populations treated in real-world settings, of which this study offers a glimpse.

Other study limitations are the potential for HIC results to have influenced the treating physicians' decisions (decision impact being a study endpoint) and evolving treatment paradigm during study timing which may complicate the interpretation of results. Since the follow-up window was only 12 months for some patients and limited to 18 months for all patients in this group, longer-term effects of the treatments studied (particularly ICI relative to chemotherapy) are limited.

Study strengths include multi-institutional real-world patient enrolment, rigorous multi-institutional test development and extensive independent validation, rapid 72-hour test turnaround, and thoroughly prespecified and documented laboratory standard operating procedures and analytical and statistical plans.

\section{CONCLUSIONS}

In conclusion, we demonstrate the potential of a proteomic test to inform treatment decisions in NSCLC independently of traditional prognostic factors in a continually evolving treatment landscape. Consideration should be given to incorporating HIC results alongside PD-L1 testing in deciding whether to treat a patient's lung cancer with ICI alone or combined with chemotherapy.

\section{Author affiliations}

${ }^{1}$ Lung Cancer, Piedmont Physicians Group, Atlanta, Georgia, USA

${ }^{2}$ Virginia Cancer Institute, Richmond, Virginia, USA

${ }^{3}$ Highlands Oncology Group, Fayetteville, Arkansas, USA

${ }^{4}$ Leo W Jenkins Cancer Center, Brody School of Medicine at East Carolina University, Greenville, North Carolina, USA

${ }^{5}$ Lewis and Faye Manderson Cancer Center at DCH Regional Medical Center,

Tuscaloosa, Alabama, USA

${ }^{6}$ Southeastern Medical Oncology Center, Goldsboro, North Carolina, USA

${ }^{7}$ Pontchartrain Cancer Center, Covington, Louisiana, USA

${ }^{8}$ The Center for Cancer and Blood Disorders, Fort Worth, Texas, USA

${ }^{9}$ St. Bernards Hospital, Inc, Jonesboro, Arkansas, USA

${ }^{10}$ Christus Saint Michael Health System, Texarkana, Texas, USA

${ }^{11}$ Christus Cancer Treatment Center, Shreveport, Louisiana, USA

${ }^{12} \mathrm{St}$. Frances Cabrini Hospital Cancer Center, Alexandria, Louisiana, USA

${ }^{13}$ Florida Precision Oncology, Division of Genesis Care, Aventura, Florida, USA

${ }^{14}$ Lynn Clinical Research Institute, Boca Raton, Florida, USA

${ }^{15}$ Essex Oncology Group, Belleville, New Jersey, USA

${ }^{16}$ Clearview Cancer Institute, Huntsville, Alabama, USA

${ }^{17}$ Clinical Research Alliance, Caremount Medical, Mount Kisco, New York, USA

${ }^{18}$ Northwest Medical Specialties, Puyallup, Washington, USA

${ }^{19}$ Hematology and Oncology Associates, Inc, Canton, Ohio, USA

${ }^{20}$ John Peter Smith Hospital, Fort Worth, Texas, USA

${ }^{21}$ Pearlman Cancer Center, Valdosta, Georgia, USA

${ }^{22}$ Edward-Elmhurst Health, Naperville, Illinois, USA

${ }^{23}$ Lake City Cancer Care, Lake City, Florida, USA

${ }^{24}$ Phelps County Regional Medical Center, Rolla, Missouri, USA

${ }^{25}$ Biodesix Inc, Boulder, Colorado, USA

${ }^{26}$ Huntsman Cancer Institute Cancer Hospital, Salt Lake City, Utah, USA 
Acknowledgements We are grateful to all the patients and investigators taking part in this study and their families. The authors also acknowledge Rachel Hartfield, $\mathrm{PhD}$, for manuscript preparation support that was funded by Biodesix, Inc. and Joanna Roder, PhD (employee of Biodesix, Inc.) for statistical interpretation assistance.

Contributors All authors contributed to study investigation, analysis and interpretation of results, and to writing and critical review of the manuscript.

Funding Biodesix sponsored the study and supported sample handling, data analysis, and manuscript preparation.

Competing interests PRW: Speaker's Bureau (Genentech), Consultant (Biodesix), Ownership/stock interest/employment (Circulogene, Theranostics). D0: Speaker's Bureau (Bayer), Consultant (Coherus, Abbvie), Ownership/stock interest (Karyopham), Honorarium (Daiichi-Sankyo, Rigel), Contracted/supported research grant (Helsinn, MEl, FibroGen). RP: Consultant (AstraZeneca, Tesaro, Amgen, Roche), Honorarium (Cardinal Health), Contracted/supported research grant (E.R. Squibb Sons, Gilead Sciences, Takeda, AstraZeneca, Genentech, Roche, Janssen, Celgene, Lilly). ESS: Speaker's Bureau (Biodesix; Caris SL; Paradigm Dx; Guardant Health; Genentech; Pfizer; Amgen; Novartis; Boehringer-Ingelheim; Merck; AstraZeneca; Dova; Sanofigenzyme, Oncocyte, Eli Lilly), Consultant (Astra Zeneca, Lilly, BluePrint Medicine, Inivata/Neogenomics). JM0: Speaker's Bureau (Pfizer-Padcev, Astellas, Xtandi), Advisory Board (Janssen-Zytiga, Pfizer-Padcev). EP: Speaker's Bureau (TG Therapeutics), Advisory Board (Flatiron Health), Consultant (Eli Lilly; Eisai). BG: Speaker's Bureau (Boehrenger Ingelheim), Advisory Board (GlaxoSmithKline). LT: Employment (Biodesix). WA: Consultant (Calithera Biosciences, AstraZeneca), Contracted/supported research grant (AstraZeneca, Bristol-Myers Squibb, Loxo).

Patient consent for publication Consent obtained directly from patient(s)

Ethics approval All enrolled subjects provided informed consent and the study was approved by Institutional Review Boards at each site.

Provenance and peer review Not commissioned; externally peer reviewed.

Data availability statement All data relevant to the study are included in the article or uploaded as online supplemental information.

Supplemental material This content has been supplied by the author(s). It has not been vetted by BMJ Publishing Group Limited (BMJ) and may not have been peer-reviewed. Any opinions or recommendations discussed are solely those of the author(s) and are not endorsed by BMJ. BMJ disclaims all liability and responsibility arising from any reliance placed on the content. Where the content includes any translated material, BMJ does not warrant the accuracy and reliability of the translations (including but not limited to local regulations, clinical guidelines, terminology, drug names and drug dosages), and is not responsible for any error and/or omissions arising from translation and adaptation or otherwise.

Open access This is an open access article distributed in accordance with the Creative Commons Attribution Non Commercial (CC BY-NC 4.0) license, which permits others to distribute, remix, adapt, build upon this work non-commercially, and license their derivative works on different terms, provided the original work is properly cited, appropriate credit is given, any changes made indicated, and the use is non-commercial. See http://creativecommons.org/licenses/by-nc/4.0/.

\section{REFERENCES}

1 Siegel RL, Miller KD, Jemal A. Cancer statistics, 2018. CA Cancer J Clin 2018;68:7-30.

2 Singal G, Miller PG, Agarwala V, et al. Association of patient characteristics and tumor genomics with clinical outcomes among patients with non-small cell lung cancer using a Clinicogenomic database. JAMA 2019;321:1391-9.

3 Nadal E, Massuti B, Dómine M, et al. Immunotherapy with checkpoint inhibitors in non-small cell lung cancer: insights from long-term survivors. Cancer Immunol Immunother 2019;68:341-52.

4 Hanna NH, Schneider BJ, Temin S, et al. Therapy for stage IV nonsmall-cell lung cancer without driver alterations: ASCO and $\mathrm{OH}$ (CCO) joint guideline update. J Clin Oncol 2020;38:1608-32.

5 National Comprehensive Cancer N. NCCN clinical practice guidelines in oncology: non-small cell lung cancer, version 3.2020 2020.

6 Camidge DR, Doebele RC, Kerr KM. Comparing and contrasting predictive biomarkers for immunotherapy and targeted therapy of NSCLC. Nat Rev Clin Oncol 2019;16:341-55.

7 Garon EB, Hellmann MD, Rizvi NA, et al. Five-year overall survival for patients with advanced Non-Small-Cell lung cancer treated with pembrolizumab: results from the phase I KEYNOTE-001 study. J Clin Oncol 2019;37:JCO1900934.
8 Brahmer JR, Rodriguez-Abreu D, Robinson AG, et al. LBA51 KEYNOTE-024 5-year OS update: First-line (1L) pembrolizumab (pembro) vs platinum-based chemotherapy (chemo) in patients (pts) with metastatic NSCLC and PD-L1 tumour proportion score (TPS) $\geq 50 \%$. Ann Oncol 2020;31:S1181-2.

9 da Veiga CRP, da Veiga CP, Drummond-Lage AP. Concern over cost of and access to cancer treatments: a meta-narrative review of nivolumab and pembrolizumab studies. Crit Rev Oncol Hematol 2018;129:133-45.

10 Taguchi F, Solomon B, Gregorc V, et al. Mass spectrometry to classify non-small-cell lung cancer patients for clinical outcome after treatment with epidermal growth factor receptor tyrosine kinase inhibitors: a multicohort cross-institutional study. J Natl Cancer Inst 2007;99:838-46.

11 Gregorc V, Novello S, Lazzari C, et al. Predictive value of a proteomic signature in patients with non-small-cell lung cancer treated with second-line erlotinib or chemotherapy (PROSE): a biomarker-stratified, randomised phase 3 trial. Lancet Oncol 2014;15:713-21.

12 Carbone DP, Ding K, Roder $\mathrm{H}$, et al. Prognostic and predictive role of the VeriStrat plasma test in patients with advanced non-small-cell lung cancer treated with erlotinib or placebo in the $\mathrm{NCIC}$ clinical Trials Group BR.21 trial. J Thorac Oncol 2012;7:1653-60.

13 Leal TA, Argento AC, Bhadra K, et al. Prognostic performance of proteomic testing in advanced non-small cell lung cancer: a systematic literature review and meta-analysis. Curr Med Res Opin 2020;36:1-9.

14 Milan E, Lazzari C, Anand S, et al. SAA1 is over-expressed in plasma of non small cell lung cancer patients with poor outcome after treatment with epidermal growth factor receptor tyrosine-kinase inhibitors. J Proteomics 2012;76:91-101.

15 Roder J, Maguire L, Georgantas R, et al. Explaining multivariate molecular diagnostic tests via Shapley values. BMC Med Inform Decis Mak 2021;21:211.

16 Fidler MJ, Fhied CL, Roder J, et al. The serum-based VeriStrat ${ }^{\circledR}$ test is associated with proinflammatory reactants and clinical outcome in non-small cell lung cancer patients. BMC Cancer 2018;18:310.

17 Grigorieva J, Asmellash S, Net L, et al. Mass spectrometry-based multivariate proteomic tests for prediction of outcomes on immune checkpoint blockade therapy: the modern analytical approach. Int $J$ Mol Sci 2020;21 doi:10.3390/ijms21030838

18 Grigorieva J, Asmellash S, Oliveira C, et al. Application of protein set enrichment analysis to correlation of protein functional sets with mass spectral features and multivariate proteomic tests. Clin Mass Spectrom 2020;15:44-53.

19 Lee J, Beatty GL. Serum amyloid A proteins and their impact on metastasis and immune biology in cancer. Cancers 2021;13 doi:10.3390/cancers 13133179

20 Ravindranathan D, Master VA, Bilen MA. Inflammatory markers in cancer immunotherapy. Biology 2021;10 doi:10.3390/ biology 10040325

21 Rossi J-F, Lu ZY, Massart C, et al. Dynamic immune/inflammation precision medicine: the good and the bad inflammation in infection and cancer. Front Immunol 2021:12:595722.

22 Di Noia V, D'Argento E, Pilotto S, et al. Blood serum amyloid A as potential biomarker of pembrolizumab efficacy for patients affected by advanced non-small cell lung cancer overexpressing PD-L1: results of the exploratory "FoRECATT" study. Cancer Immunol Immunother 2021;70:1583-92.

23 Rich P, Roder J, Dubay J, et al. Real-world performance of bloodbased proteomic profiling in frontline immunotherapy treatment in advanced stage NSCLC. Int J Radiat Oncol Biol Phys 2019;104:236.

24 Grossi F, Rijavec E, Biello F, et al. P3.02c-074 evaluation of a pretreatment serum tests for nivolumab benefit in patients with nonsmall cell lung cancer. J Thor Oncol 2017;12:S1322.

25 Mellert H, Foreman T, Jackson L, et al. Development and clinical utility of a blood-based test service for the rapid identification of actionable mutations in non-small cell lung carcinoma. J Mol Diagn 2017;19:404-16.

26 Lazzari C, Spreafico A, Bachi A, et al. Changes in plasma massspectral profile in course of treatment of non-small cell lung cancer patients with epidermal growth factor receptor tyrosine kinase inhibitors. J Thorac Oncol 2012;7:40-8.

27 Cogdill AP, Andrews MC, Wargo JA. Hallmarks of response to immune checkpoint blockade. Br J Cancer 2017;117:1-7.

28 Waterhouse D, Lam J, Betts KA, et al. Real-world outcomes of immunotherapy-based regimens in first-line advanced non-small cell lung cancer. Lung Cancer 2021;156:41-9.

29 Dudnik E, Moskovitz M, Rottenberg Y, et al. Pembrolizumab as a monotherapy or in combination with platinum-based chemotherapy in advanced non-small cell lung cancer with PD-L1 tumor 
proportion score (TPS) $\geq 50 \%$ : real-world data. Oncoimmunology 2021;10:1865653.

30 Cramer-van der Welle CM, Verschueren MV, Tonn M, et al. Real-world outcomes versus clinical trial results of immunotherapy in stage IV non-small cell lung cancer (NSCLC) in the Netherlands. Sci Rep 2021;11:6306.

31 Walker PR, Schaefer E, Thompson Rich P, et al. PS06.03 insight study interim analysis: impact of VeriStrat results on patient management in early and locally advanced NSCLC. Journal of Thoracic Oncology 2017;12:S1582-3.

32 Reck M, Rodríguez-Abreu D, Robinson AG, et al. Pembrolizumab versus chemotherapy for PD-L1-positive non-small-cell lung cancer. N Engl J Med 2016;375:1823-33.

33 Mok TSK, Wu Y-L, Kudaba I, et al. Pembrolizumab versus chemotherapy for previously untreated, PD-L1-expressing, locally advanced or metastatic non-small-cell lung cancer (KEYNOTE-042): a randomised, open-label, controlled, phase 3 trial. Lancet 2019;393:1819-30.

34 Carbone DP, Reck M, Paz-Ares L, et al. First-line nivolumab in stage IV or recurrent non-small-cell lung cancer. N Engl J Med 2017;376:2415-26.
35 Gandhi L, Rodríguez-Abreu D, Gadgeel S, et al. Pembrolizumab plus chemotherapy in metastatic non-small-cell lung cancer. $N$ Engl J Med 2018;378:2078-92.

36 Paz-Ares L, Luft A, Vicente D, et al. Pembrolizumab plus chemotherapy for squamous non-small-cell lung cancer. $N$ Engl $J$ Med 2018;379:2040-51.

$37 \mathrm{Kim} \mathrm{R,} \mathrm{Keam} \mathrm{B,} \mathrm{Hahn} \mathrm{S,} \mathrm{et} \mathrm{al.} \mathrm{First-line} \mathrm{pembrolizumab} \mathrm{versus}$ pembrolizumab plus chemotherapy versus chemotherapy alone in non-small-cell lung cancer: a systematic review and network metaanalysis. Clin Lung Cancer 2019;20:331-8.

38 Dafni U, Tsourti Z, Vervita K, et al. Immune checkpoint inhibitors, alone or in combination with chemotherapy, as first-line treatment for advanced non-small cell lung cancer. A systematic review and network meta-analysis. Lung Cancer 2019;134:127-40.

39 Liu J, Li C, Seery S, et al. Identifying optimal first-line interventions for advanced non-small cell lung carcinoma according to PDL1 expression: a systematic review and network meta-analysis. Oncoimmunology 2020;9:1746112.

40 Roxburgh CSD, McMillan DC. Cancer and systemic inflammation: treat the tumour and treat the host. Br J Cancer 2014;110:1409-12.

41 Chen DS, Mellman I. Elements of cancer immunity and the cancerimmune set point. Nature 2017;541:321-30. 